\title{
Interactive comment on "Instrument observation strategy of new generation three-axis stabilized geostationary meteorological satellite of China" by Jian Shang et al.
}

Jian Shang et al.

shangjian@cma.gov.cn

Received and published: 8 May 2019

The manuscript has been revised earnestly according to the reviewer's suggestions. Thanks very much for the detailed guidance. The main modifications are listed below, and the revised manuscript is also submitted. Thank you again.

Question 1: Please add an introduction of the instruments to help the readers to understand the background, including the bands and resolution.

Answer: Geostationary imager is the most effective instrument onboard the geostationary satellites all over the world. AGRI is the core instrument of FY-4A satellite, 
which aims to carry out high temporal and spatial resolution imaging of the atmosphere, cloud, land and ocean, in over 14 spectral bands in visible (VIS), near infrared (NIR) and infrared (IR) spectral regions, providing important information for weather analysis and forecast, climate research, environment and disaster monitoring. Main parameters of AGRI are shown is Table 1, and the information of its 14 bands is summarized in Table 2.

Interactive

Table 1: Main parameters of AGRI. (Please see the supplement.)

Table 2: Spectral configuration of AGRI. (Please see the supplement.)

GIIRS is another important instrument onboard FY-4A. Its main objective is to detect atmospheric temperature, moisture and trace gas content precisely, providing input data for numerical weather forecast, disastrous weather monitoring and atmospheric chemical composition detection. It has visible, medium / shortwave and longwave IR bands. Main parameters of GIIRS are shown in Table 4 of the manuscript.

Table 4: Main parameters of GIIRS. (Please see the supplement.)

Question 2: The detector array of star observation should be provided as auxiliary explanation, before the star observation strategies are proposed.

Answer: AGRI has 14 observation bands, the second $(0.55-0.75 \mu \mathrm{m})$ of which is designed to sense stars of magnitude higher than 6.0, as shown in Table 2. The detector size is 32 (north-south direction) by 4 (west-east direction), with a gap between adjacent columns, which is shown is Fig. 1. This must be considered in developing AGRl's star observation strategy to guide its in-orbit daily star sensing.

Figure 1: Detector array diagram of AGRI. (Please see the supplement.)

GIIRS is designed deliberately with a visible band $(0.55-0.75 \mu \mathrm{m})$ to sense stars of magnitude lower than 6.5. The detector size is 330 (north-south direction) by 256 (west-east direction) and no gap between adjacent columns as well as rows, which is shown is Fig. 3 of the manuscript. Star observation strategy of GIIRS must also be developed, con-

Printer-friendly version

Discussion paper 
sidering both the similarities and differences between AGRI and GIIRS. The similarities include requirements of star centroid extraction as well as thermal elastic deformation GID parameter calculation. The main difference comes from the different instantaneous field of view (IFOV), which will be analyzed in detail.

Figure 3: Detector array diagram of GIIRS. (Please see the supplement.)

Interactive

comment

Question 3: The star observation strategy can be analyzed based on instrument parameters and characteristics, respectively in page 5 and page 7.

Answer: For AGRI, the detector size is 32 by 4 , with a gap between adjacent columns, which is shown in Fig. 1. This must be considered in developing AGRl's star observation strategy. Dwell observation mode is recommended for star sensing, waiting for the star crossing the whole focal plane, aiming at obtaining star observation data of relative long time series. This can effectively improve the accuracy of star centroid extraction. AGRI's angular resolution determines the minimal distance between any two candidate stars. Another characteristic of AGRI is its small detector size, which means most of the time only one star can be observed. This directly influences the minimal distance between the candidate star and another star.

For GIIRS, its star sensing ability is higher than AGRI, which means there are more stars that can be observed. And its detector size is larger, as shown in Fig. 3, which means not only one star but also multiple stars can be observed at a time. Thus multiple star selection strategy should first be considered because this is advantageous for star recognition. Another characteristic of GIIRS is it can't wait for the star crossing the whole focal plane, as its detector is so large. A small area of less than 10 pixels near the center of the detector is chosen to be used for star sensing, in order to save observation time as well as to avoid the effect of distortion at the edge of the detector.

Thus two specific star observation strategies are developed respectively for the two instruments, based on their similarities and differences.

Printer-friendly version

Discussion paper 
Question 4: The overall instrument observation strategy, including full disk observation, blackbody observation, star observation, et al., should be described and summarized GID in a table or figure clearly in page 8 before the simulation.

Answer: The AGRI observation strategy and GIIRS observation strategy are proposed above in detail. In every time block, one full disk task or three China region tasks, one infrared background task, one blackbody task and one star task are arranged for AGRI, while one region task, one blackbody task, one cold space task and one star task are arranged for GIIRS. The time block is shown if Fig. 4 for clarity. FY-4A satellite's daily operation is implemented based on these two time blocks.

Figure 4: Time blocks for AGRI and GIIRS used in daily operation: (a) AGRI and (b) GIIRS. (Please see the supplement.)

Please also note the supplement to this comment:

https://www.geosci-instrum-method-data-syst-discuss.net/gi-2018-35/gi-2018-35-

AC2-supplement.zip

Interactive comment on Geosci. Instrum. Method. Data Syst. Discuss., https://doi.org/10.5194/gi-2018-35, 2019. 


\section{GID}

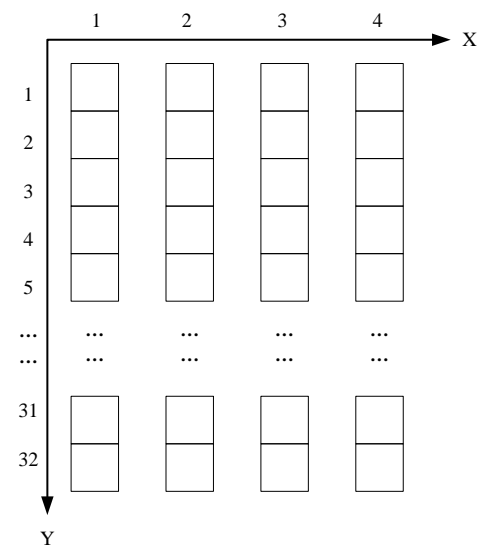

Interactive comment

Figure 1: Detector array diagram of AGRI.

Fig. 1. Detector array diagram of AGRI 


\section{GID}

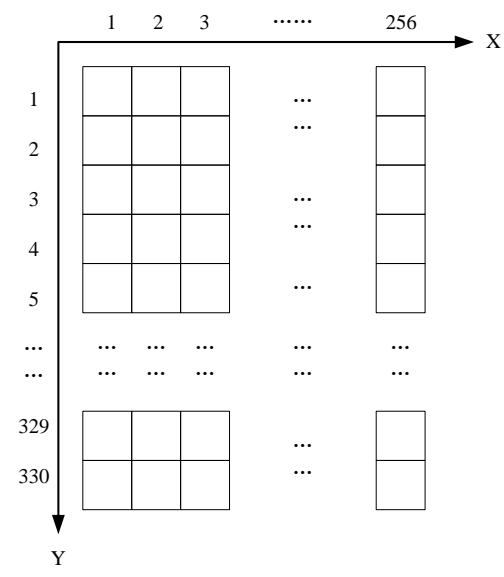

Figure 3: Detector array diagram of GIIRS.

Interactive comment

Fig. 2. Detector array diagram of GIIRS 


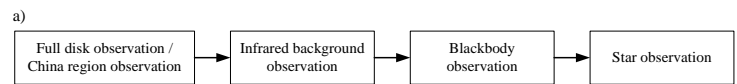

Interactive comment

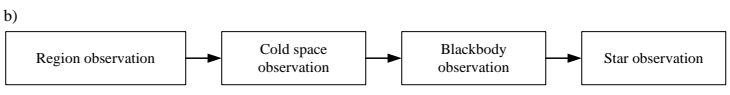

Figure 4: Time blocks for AGRI and GIIRS used in daily operation: (a) AGRI and (b) GIIRS.

Fig. 3. Time blocks for AGRI and GIIRS used in daily operation 